\title{
Methods of Synthesis of Oxides of Iron and Removing Compounds Arsenic in Water
}

\section{Métodos de Síntesis de Óxidos de Hierro y Compósitos para Remover Arsénico en Agua}

\author{
Carlos Medina ${ }^{1,2}$, Jorge Silva Y.,2, and Adrian Rodriguez ${ }^{1,2}$ \\ ${ }^{1}$ Escuela Superior Politécnica de Chimborazo, Facultad de Ciencias, Riobamba, Ecuador \\ ${ }^{2}$ Grupo de Investigaciónen Materiales Avanzados (GIMA), Facultad de Ciencias, ESPOCH
}

I International Congress of Science and Technology Morona Santiago-CICTMS 2020

Corresponding Author:

Carlos Medina

carlos.medinas@espoch.edu.ec

Published: 29 August 2021

Production and Hosting by

Knowledge E

(a) Carlos Medina et al. This article is distributed under the terms of the Creative Commons Attribution License, which permits unrestricted use and redistribution provided that the original author and source are credited.

\section{Abstract}

Environmental pollution by heavy metals has been extensively researched using different materials and techniques but, this problem has not been fully resolved. High adsorption capacity of iron oxides such as hematite, magnetite, goethite and, ferrihydrite to remove arsenic are described in this review. There are many international investigations about the minerals of iron oxides and they describe the composition, properties, synthesis methods and involved variables. Active carbon, silicones and polymers had been used to obtain composites with iron oxides and they have gave better results to remove different anions and cations. The aim in this paper is to introduce studies already carried out and encourage research in this topic to take advantage of the particular characteristics of iron oxides and use them in the environmental remediation. In addition, it is important to introduce the natural iron oxides availability that have a lot of field to study. The literature search on the subject was carried out in Science Direct and high impact articles related to natural or synthetic oxides were used.

Keywords: Arsenic, hematite, magnetite, goethite, iron composites.

\section{Resumen}

La contaminación ambiental por metales se ha estudiado mucho con diferentes materiales y técnicas, pero aún no se ha logrado resolver por completo este problema. La alta capacidad de adsorción de los óxidos de hierro como la hematita, magnetita, goetita, ferrihidrita, para la remoción de arsénico en agua son descritos en este trabajo de revisión. Existen muchas investigaciones internacionales de los óxidos de hierro en las que se expone la composición de estos minerales, las propiedades, métodos de síntesis y las variables que intervienen. El carbón activo, las siliconas y los polímeros son materiales que se han usado para formar compositos con estos óxidos que han contribuido a obtener mejores resultados en la remoción de diferentes aniones y cationes. Con este trabajo se pretende difundir estudios ya realizados e incentivar la investigación en este campo para aprovechar las características particulares de los óxidos de hierro y usarlos como remediadores ambientales. Ademas, es importante dar a conocer la existencia de óxidos de hierro naturales que dejan mucho campo por estudiar. 


\section{Introducción}

La contaminación ambiental es uno de los problemas más estudiados en la actualidad, en especial la generada por metales pesados y metaloides como el mercurio, plomo, zinc, cromo, cadmio y arsénico, debido a que han sido encontrados en todo tipo de alimentos y muestras de agua de diferente naturaleza [1].

Más de $\mathbf{2 0 0}$ millones de personas en 70 países están expuestas al arsénico a través del agua potable [2]. A nivel de Latinoamérica, se han realizado varios estudios sobre la exposición a arsénico en Chile, México, Argentina, Bolivia, Brasil, Colombia, Ecuador y Uruguay [3].

Los óxidos de hierro (óxidos, hidróxidos y oxihidróxidos), hematita $\left(\mathrm{Fe}_{2} \mathrm{O}_{3}\right)$, ferrihidrita $\left[\mathrm{Fe}_{10} \mathrm{O}_{14}(\mathrm{OH})_{2}\right]$, goethita $(\alpha-\mathrm{FeOOH})$, magnetita $\left(\mathrm{Fe}_{3} \mathrm{O}_{4}\right)$, lepidocrocita $(\gamma$-FeOOH$)$ entre otros se han estudiado ampliamente para la remoción de arsénico desde medios acuosos $[4,5]$.

En este contexto el objetivo de este artículo es revisar la capacidad de remoción de arsénico con los diferentes óxidos de hierro y los métodos de síntesis que permitan un mayor rendimiento, así mismo, dar a conocer metodologías empleadas para la funcionalización con otros materiales que permita mejorar las características de estos óxidos.

\section{Metodología}

La revisión de literatura se ha realizó por medio de Sciencedirect. Los descriptores utilizados en la búsqueda fueron: iron oxides, synthetic iron oxides and arsenic water.

La recopilación de los artículos utilizados en la revisión se efectuó entre octubre de 2019 y agosto de 2020. Se han considerado 46 artículos de los cuales se han extraído las principales ideas plasmadas en este documento.

En la primera parte se revisa la capacidad de remoción de arsénico alcanzada por diferentes óxidos de hierro y luego se revisan algunos de los métodos empleados en los procesos de síntesis de los óxidos de hierro.

\section{Desarrollo y Discusión}

\section{1. Óxidos de hierro como adsorbentes}

Corresponden a minerales que han demostrado ser eficientes para la remoción de As del agua debido a su baja solubilidad y su capacidad de adsorción siendo más efectiva la adsorción del As en su forma pentavalente que en su forma trivalente [6, 7]. Los minerales que contienen hierro se encuentran en forma de óxidos, hidróxidos y oxo (hidróxidos) tales como: hematita, magnetita, goetita, ferrihidrita, siderita, limonita, maghemita, burstita, lepidocrocita, akaganeíta, schwermanita, feroxihita y bernalita. Siendo los seis primeros, los minerales más utilizados como adsorbentes $[6,8]$ que 
generalmente, deben ser molidos y tamizados para generar partículas con áreas superficiales grandes, por ejemplo, Gimenez et al. (2007) utilizó hematita y magnetita de diferente tamaño para remover contaminantes de agua y encontró que las partículas de menor tamaño remueven más cantidad [8].

\subsubsection{Hematita}

La hematita $\left(\mathrm{Fe}_{2} \mathrm{O}_{3}\right)$ tiene una estructura de tipo hexagonal que contiene a los átomos de oxígeno unidos a los cationes en una coordinación octaédrica [8]. Torrent et al. (1994) estudiaron una variedad de hematitas en las que ninguna tenía una composición homogénea, sino que variaban en el contenido de hematita, sílice y goetita. Sus resultados también indicaron que los cristales de hematita se encuentran predispuestos en una cara prismática de forma elongada. Por ejemplo se ha reportado hematita con la siguiente composición $64.9 \% \mathrm{Fe}, 0.52 \% \mathrm{Al}_{2} \mathrm{O}_{3}, 3.95 \% \mathrm{SiO}_{2}$ y $0.16 \% \mathrm{MnO}$ con un área superficial de $20.53 \mathrm{~m}^{2} / \mathrm{g}$ [8]. Por otro lado, también se reportan minerales con una composición expresada de la siguiente forma: $86 \%$ hematita, $4 \%$ siderita, $5 \%$ goetita, $1 \%$ moscovita y el restante de cuarzo. Este mineral fue estudiado para la adsorción de As alcanzando una remoción de alrededor $75 \%$ de As (V) y $35 \%$ de As (III). Según los autores, esto puede haber ocurrido debido a la estructura estratificada del mineral que contiene varias micro fisuras. Además, realizaron tambien experimentos con diferentes tamaños de partículas, obteniendo una mayor remoción con un tamaño $<0.25 \mathrm{~mm}$ cundo se trabajó con dosis de $40 \mathrm{~g}$ adsorbente/L [9]. Así mismo, cuando utilizaron hematita con un tamaño de partícula menor a $100 \mu \mathrm{m}$, su área superficial fue de $4 \mathrm{~m}^{2} / \mathrm{g}$ [10].

Gimenez et al. (2007) estudiaron la adsorción de As (III) y As (V) en hematita, magnetita y goetita natural y las compararon con adsorbentes sintéticos. Sus resultados indican que la hematita adsorbió una mayor cantidad de As que los otros dos minerales ya que estos últimos se saturaron más rápidamente. Además se estudió la variación de la concentración del arsénico eliminado de las soluciones usando los tres diferentes absorbentes por separado y con tres variables de estudio ( $\mathrm{pH}$ de solución, tamaño de partícula y dosis del adsorbente). La principal tendencia observada para la remoción del contaminante cuando se varía el pH fue la disminución de la sorción en los tres sorbentes a valores de $\mathrm{pH}$ alcalino. Observaron mayor remoción de As (III) en la superficie de la hematita en todo el rango de $\mathrm{pH}$ en comparación con la goetita y la magnetita. Los adsorbentes naturales (minerales) y adsorbentes sintéticos estudiados en su investigación tuvieron capacidades de adsorción semejantes para los dos tipos de arsénico [11].

Aredes et al. (2013) emplearon $0.5 \mathrm{~g}$ de hematita por cada $100 \mathrm{~mL}$ de muestra a tratar. El mineral fue capaz de adsorber un $20 \%$ de As (V) de una concentración inicial de 5 ppm. Cuando la dósis del adsorbente se aumentó a $10 \mathrm{~g}$, el mineral fue capaz de remover todo el As (V) de la muestra, aunque se pudo haber formado un precipitado en la superficie de los adsorbentes [12]. El estudio realizado por Zhang et al. (2004) ocupó hematita variando el $\mathrm{pH}$ de las soluciones desde 4.5 a 6.5 y una dosis de adsorbente de $5 \mathrm{~g} / \mathrm{L}$. Este mineral redujo la concentración de $A s(\mathrm{~V})$ en un 99\%. Así 
mismo, presencia de sílice y fósforo redujeron la capacidad de adsorción del mineral mientras que sulfatos y cloruros la mejoraron [13]. El proceso de adsorción de As (V) con hematita ha demostrado ser representado por una Isoterma de Langmuir. En el caso del pH la adsorción de As (V) es favorecida a un pH de 4.2 a 7.1. En dicho estudio se utilizó una solución de arsénico de concentración 1000 ppb, en el cual la hematita con un área superficial de $14.40 \mathrm{~m}^{2} / \mathrm{g}$ tuvo una capacidad de adsorción de $0.20 \mathrm{mg} / \mathrm{g}$ [14].

\subsubsection{Magnetita}

La magnetita $\left(\mathrm{Fe}_{3} \mathrm{O}_{4}\right)$ se encuentra generalmente en forma de cristales octaédricos y puede ser transformada en hematita o goetita. La composición de los cristales es generalmente $22.4 \% \mathrm{Fe}$ y $27.6 \% \mathrm{O}$ [8]. Las propiedades magnéticas de este mineral facilitan la separación del adsorbente del contaminante. La producción de nano partículas de magnetita ha sido útil para la adsorción de arsénico en sus formas más comunes siendo el cambio de tamaño de $300 \mathrm{~nm}$ a $12 \mathrm{~nm}$ el factor más importante para incrementar la efectividad de las nano partículas en 200 veces [15].

\subsubsection{Goetita}

La goetita $(\alpha-\mathrm{FeOOH})$ tiene cristales en forma de agujas y su estructura posee bandas de $\mathrm{FeO}(\mathrm{OH})$ octaédricas que comparten vértices y bordes formando un túnel octaédrico de 2'1 que se encuentra enlazado parcialmente por enlaces de hidrógeno. Su composición se estima en la siguiente: $62.9 \% \mathrm{Fe}, 27 \% \mathrm{O}$ y $10.1 \%$ de $\mathrm{H}_{2} \mathrm{O}$ [8]. Mamindy-Pajany et al. (2009) utilizó adsorbentes de magnetita (área superficial $1.60 \mathrm{~m}^{2} / \mathrm{g}$ y punto isoeléctrico de 6.40), goetita (área superficial $=11.61 \mathrm{~m}^{2} / \mathrm{g}$ y punto isoeléctrico de 6.9) y hematita (área superficial $=1.66 \mathrm{~m}^{2} / \mathrm{g}$ y punto isoeléctrico de 8.1). Partiendo de $50 \mathrm{~mL}$ de una solución de $100 \mathrm{ug} / \mathrm{L}$ de $\mathrm{As}(\mathrm{V})$ y $0.2 \mathrm{~g}$ de adsorbente, los tres adsorbentes removieron el $100 \%$ del As (V); hematita alcanzo esta cantidad en un $\mathrm{pH}$ : 2-11, mientras que los otros dos adsorbentes lo alcanzaron en un $\mathrm{pH}$ : 2-8 [16]. Este factor de $\mathrm{pH}$ también fue observado por Mohan y Pittman (2007), cuyos resultados fueron óptimos a un pH de 5.0. La goetita en este estudio tenía un tamaño de $0.01 \mathrm{~mm}$ y un área superficial de $11.61 \mathrm{~m}^{2} / \mathrm{g}$. Se partió de una concentración de arsénico de $495 \mathrm{ppb}$ y la goetita tuvo una capacidad de adsorción de $0.500 \mathrm{mg} / \mathrm{g}$ [17].

\subsubsection{Otros}

La siderita $\left(\mathrm{FeCO}_{3}\right)$ se encuentra mezclada con arcillas como capas concéntricas. La limonita $\left(\mathrm{FeOOH} . \mathrm{nH}_{2} \mathrm{O}\right)$ al igual que la siderita son minerales de los cuales su composición y forma todavía atraen cierto debate. La ferrihidrita se forma como precipitado de bacterias que fijan hierro o por la hidrolisis natural de sales de hierro y se puede utilizar para la formación de otros minerales de hierro. A un $\mathrm{pH}$ de 7, una ferrihidrita fue capaz de adsorber $87.6 \mathrm{mg}$ de As (V) por $1 \mathrm{~g}$ de adsorbente de una solución $150 \mathrm{ppm}$ como concentración inicial de arsénico [18]. Guo et al. (2007) utilizaron varias sideritas 
para adsorber As; la más efectiva tenía una composición de 78\% siderita, 1\% calcopirita, $19 \%$ goetita y $1 \%$ cuarzo alcanzando una remoción de alrededor de $70 \%$ de As (V), $20 \%$ de As (II). Las condiciones experimentales fueron las siguientes: concentración As: 1000 ppb, tamaño de partícula: $0.25-0.5 \mathrm{~mm}$, carga de adsorbente: $10 \mathrm{~g} / \mathrm{L}$, tiempo de contacto: 1440 min y temperatura: $20^{\circ} \mathrm{C}$ [19]. En el caso de limonita, un estudio demostró la capacidad de este mineral para remover $900 \mathrm{mg}$ de $\mathrm{As}(\mathrm{V})$ por cada $\mathrm{kg}$ de mineral [20].

\subsection{Métodos de síntesis de óxidos y compósitos de óxidos de hierro}

Los óxidos de hierro en su gran mayoría son sintetizados como nano-estructuras de diferentes formas. Estos luego pueden ser soportados sobre otros materiales como carbón activado, siliconas, celulosa y otros materiales orgánicos formando así con ellos los llamados compositos. Se conoce de una gran variedad de métodos de síntesis tales como microemulsión, pirolisis por pulverización con llama, decapado, ablación laser, técnicas de deposición química, condensación, síntesis de sol-gel, reducción química, co-precipitación, etc.

Todos estos nano-materiales pueden ser caracterizados por técnicas como termogravimetría (TGA), microscopía de barrido electrónico (SEM), espectroscopía infrarroja (IR), Difracción de Rayos-X (XDR), microscopía electrónica de transmisión (TEM), adsorción de $\mathrm{N}_{2}$, etc.

\subsubsection{Goetita}

Es uno de los óxidos de hierro térmicamente más estables, se ha estudiado su capacidad de adsorción debido a su gran área superficial que permite adsorber gases, aniones y cationes orgánicos e inorgánicos, pero en especial se ha investigado con metales pesados [21, 22]. Aunque la mayoría de los métodos de síntesis de goetita requieren condiciones severas y mezclas complejas de reactivos, se han estudiado vías más sencillas para obtenerlo. Para sintetizar por el método de precipitación por ejemplo, se mezcla cloruro férrico y amonio, el precipitado se separa por centrifugación y es lavado varias veces con varios reactivos; luego purificado y aislado. La nanopartículas obtenidas tienen forma cúbica y dieron buena estabilidad en sus estudios [23]. El tratamiento hidro-térmico a $298^{\circ} \mathrm{C}$ de la goetita obtenida por suspensión de hidróxido férrico amorfo gracias a la mezcla de nitrato férrico nano hidratado [ $\left.\mathrm{Fe}\left(\mathrm{NO}_{3}\right)_{3} \cdot 9 \mathrm{H}_{2} \mathrm{O}\right]$ con hidróxido sódico $(\mathrm{NaOH})$ es otra vía para sintetizar cristales de goetita, aunque no resultaron nanopartículas totalmente puras ya que se evidenció presencia de nitrato de sodio formado en la reacción [24]. Se puede también obtener este nanomaterial impregnado en varios soportes como óxidos de grafeno [25], celulosa [26], copolímero de metacrilato de glicidilo [27], poliacrilamida [28], todos estos compositos fueron estudiados para la remoción de metales pesados en aguas de diferente procedencia. 


\subsubsection{Magnetita (Fe304)}

La propiedad de mayor importancia de la magnetita es el ferromagnetismo, que depende del tamaño $[29,30]$ y la forma de las partículas que se haya obtenido en la síntesis. El método más sencillo de síntesis es la precipitación por reacción de sulfato ferroso heptahidratado $\left(\mathrm{FeSO}_{4} \cdot 7 \mathrm{H}_{2} \mathrm{O}\right)$ con hidróxido sódico $(\mathrm{NaOH})$, el precipitado se lo deshidrata a temperaturas relativamente bajas $\left(50^{\circ} \mathrm{C}\right)$, el $\mathrm{pH}$ es un parámetro de control crítico ya que a pH 10 se obtiene magnetita y a pH 4 se genera goetita [31]. La síntesis de un composito de magnetita/quitosano dependiendo de los parámetros de reacción, la morfología de las nanopartículas obtenidas varía desde esféricas, forma de semilla de arroz hasta forma de grumos, todo esto usando cloruro de hierro como precursor y urea como agente precipitante. El tiempo de reacción es el que determinó la morfología, pues en $10 \mathrm{hr}$ de reacción se obtuvieron nanopartículas esféricas, mientras que en $50 \mathrm{hr}$ de reacción generaron nanopartículas con forma de semilla de arroz [32]. Se ha estudiado por otra parte en el campo ambiental para eliminación fotocatalítica de compuestos orgánicos como nitrobenceno [33] e isatina [25]. Así mismo, se ha estudiado la remoción de múltiples cationes $\left(\mathrm{Cr}^{3+}, \mathrm{Cr}^{6+}, \mathrm{Cd}^{2+}, \mathrm{Cu}^{2+}, \mathrm{Ni}^{2+}, \mathrm{Zn}^{2+}\right)$ aprovechando el magnetismo de la magnetita [34] y aniones $\left(\mathrm{SO}_{4}{ }^{2-}, \mathrm{Cr}_{2} \mathrm{O}_{7}{ }^{2-}, \mathrm{AsO}_{4}{ }^{3-}\right)$ [35-37], entre otros. Existe estudios en una gran variedad de soportes para elaborar compositos con la magnetita, entre los soportes más usuales se destaca la gelatina [38], celulosa [39], siliconas [40] y óxidos de grafeno [41].

\subsubsection{Maghemita $(\gamma-\mathrm{Fe} 2 \mathrm{O} 3)$}

La maghemita es un material de mucha importancia en varios campos de aplicación como materiales nano estructurados a manera de nanoparticulas, nanotubos, nanobarras, nanoalambres o nanolaminas [42]. Uno de los métodos menos complejos para su obtención es por descomposición térmica de citrato férrico a elevadas temperaturas que pueden ir desde los 300 a $350^{\circ} \mathrm{C}$ por aproximadamente $2 \mathrm{hr}$ y al superar esta temperatura de calcinación, se ha descubierto que predomina la formación de una fase de hematita [43]. Otro método de preparación es a partir de nitrato férrico mezclado con solución de trietilentetramina y calentamiento a $450^{\circ} \mathrm{C}$, el material obtenido es la maghemita que finalmente se lo tritura, lava con peróxido de hidrógeno para eliminar el carbón residual y se vuelve a secar a bajas temperaturas obteniendo como producto un nanopolvo de maghemita superparamagnético [44]. Otro sencillo método es la mezcla de nitrato férrico con urea disueltos en una mezcla etilenglicol-agua y calentamiento a $100^{\circ} \mathrm{C}$, el precipitado recolectado se lava hasta $\mathrm{pH}$ neutro y se lo seca para finalmente calcinarlo a $230^{\circ} \mathrm{C}$. Sin la adición del etilenglicol, obtuvieron hematita nanoesférica mientras que la adición del etilenglicol promovió la formación de otras nano estructuras, demostrando con esto la importancia de este disolvente. 


\subsubsection{Ferrihidrita (5Fe2O3.9H2O)}

La ferrihidrita es un oxihidróxido de hierro pobremente cristalino que precipita en manantiales cargados de hierro, formado como precursor de los óxidos de hierro más estables, como la magnetita y la hematita. Una manera de obtenerlo soportado sobre sílice es por mezcla de soluciones de nitrato férrico o sulfato férrico con sílice en presencia de hidróxido de sodio y calentados a $85^{\circ} \mathrm{C}$. Tanto nitrato como sulfato producen ferrihidrita pero la precipitación de sílice se produce mucho más rápidamente en medios de nitrato férrico que en sulfato férrico [45]. Otro método sencillo consiste en mezclar hidróxido de sodio con cloruro férrico y posteriormente secarlo a temperatura ambiente para obtener ferrihidrita formando grupos de partículas agregadas llamados clusters con forma de pudin de ciruela [46].

\section{Conclusion}

La presencia de arsénico en fuentes de agua es un problema frecuente en la actualidad. Los óxidos de hierro como se ha visto son una alternativa para eliminar este contaminante, sin embargo, es necesario investigar los diferentes parámetros de síntesis con los métodos ya existentes como la temperatura, el tiempo de reacción o la concentración de los precursores para obtener compuestos más puros o de morfologías únicas, ya que en algunos estudios se obtiene mezclas de los diferentes óxidos. De igual manera, estudiar nuevos compositos que permitan desarrollar propiedades y mejores caracteristicas para alcanzar mayores capacidades de remoción de los dos tipos de arsénico tratados en este documento, así como, otros metales presentes en el agua. Las propiedades magnéticas de algunos de los óxidos de hierro facilitan la separación de los metales del agua, por esta razón, se podría investigar la fucionalización de la magnetita por ejemplo con materiales adsorbentes como carbón activado, seolitas, siliconas, etc., que posiblemente mejoren la eficiencia de la remoción de los metales.

\section{Agradecimientos}

A la Escuela Superior Politécnica de Chimborazo.

Al Instituto de Investigaciones de la Escuela Superior Politécnica de Chimborazo.

\section{Conflicto de Intereses}

No existen intereses particulares por parte de los autores o de la entidad científica que pudiesen afectar directa o indirectamente a los resultados.

\section{References}

[1] Quansah R, Armah FA, Essumang DK et al. Association of arsenic with adverse pregnancy outcomes/ infant mortality. Enviromental Heal Perspect. 2015;123(5):412-22. 
[2] Minatel BC, Sage AP, Anderson C et al. Environmental arsenic exposure: From genetic susceptibility to pathogenesis. Environ Int. 2018;112:183-97.

[3] Khan KM, Chakraborty R, Bundschuh J, Bhattacharya P, Parvez F. Health effects of arsenic exposure in Latin America: An overview of the past eight years of research. Sci Total Environ. 2020;710:136071.

[4] Hao L, Liu M, Wang N, Li G. A critical review on arsenic removal from water using iron-based adsorbents. RSC Adv. 2018;8(69):39545-60.

[5] Polowczyk I, Cyganowski P, Ulatowska J, Sawiński W, Bastrzyk A. Synthetic iron oxides for adsorptive removal of arsenic. Water Air Soil Pollut. 2018;229(6):1-10.

[6] Elisabeth A. Retención de contaminantes metálicos y arsénico por oxo (hidr) óxidos de hierro puros y sustituídos. [Doctoral thesis]. Buenos Aires: Facultad de Ciencias Exactas y Naturales, Universidad de Buenos Aires; 2013.

[7] Magnesio CONÓDE. Adosrción de arsénico en zeolita natural pretratada con oxidos de magnesio. Rev Int Contam Ambient. 2009;25(4):217-27.

[8] Journal Al, Gallegos-garcia M, Ramírez-muñiz K, Song S. Arsenic removal from water by adsorption using iron oxide minerals as adsorbents: A review. Miner Process Extr Metall Rev An Int J. 2012;7508(May).

[9] Giles DE, Mohapatra M, Issa TB, Anand S, Singh P. Iron and aluminium based adsorption strategies for removing arsenic from water. J Environ Manage. 2011;92(12):3011-22.

[10] Rabung T, Geckeis H, Kim J, Beck HP. Sorption of Eu (III) on a Natural Hematite: Application of a Surface Complexation Model. 1998;161:153-61.

[11] Gim J, Pablo J De, Rovira M, Duro L. Arsenic sorption onto natural hematite, magnetite, and goethite. 2007;141:575-80.

[12] Aredes S, Klein B, Pawlik M. The removal of arsenic from water using natural iron oxide minerals q. J Clean Prod. 2013;60:71-6.

[13] Zhang W, Singh P, Paling E, Delides S. Arsenic removal from contaminated water by natural iron ores. Miner Eng. 2004;17:517-24.

[14] Evier ELS. Adsorption technique for the treatment of As (V) -rich effluents. Colloids Surfaces $A$ ehysicochemical Eng Asp. 1996;

[15] Mayo JT, Yavuz C, Yean S et al. The effect of nanocrystalline magnetite size on arsenic removal. Sci Technol Adv Mater. 2017;6996(October).

[16] Mamindy-pajany Y, Hurel C, Marmier N. Arsenic adsorption onto hematite and goethite. C R Chim. 2009;12:876-81.

[17] Mohan D, Pittman CU. Arsenic removal from water/wastewater using adsorbents - A critical review. J Hazard Mater. 2007;142:1-53.

[18] Lafferty BJ, Loeppert RH. Methyl Arsenic Adsorption and Desorption Behavior on Iron Oxides. Environ Sci Technol. 2005;39(7):2120-7.

[19] Guo H, Stu D, Berner Z. Removal of arsenic from aqueous solution by natural siderite and hematite. Appl Geochemistry. 2007;22:1039-51.

[20] Viet $\mathrm{PH}$, Con $\mathrm{TH}$, Ha CT et al. Investigation of arsenic removal technologies for drinking water in Vietnam. Arsen Expo Heal Eff V. 2003;459-69.

[21] Liu H, Chen T, Frost RL. An overview of the role of goethite surfaces in the environment. Chemosphere. 2014;103:1-11.

[22] Jaiswal A, Banerjee S, Mani R, Chattopadhyaya MC. Synthesis, characterization and application of goethite mineral as an adsorbent. Biochem Pharmacol. 2013;1(3):281-9.

[23] Lunin A V, Kolychev EL, Mochalova EN, Cherkasov VR, Nikitin MP. Synthesis of highly-specific stable nanocrystalline goethite-like hydrous ferric oxide nanoparticles for biomedical applications by simple precipitation method. J Colloid Interface Sci. 2019;541:143-9.

[24] Nørlund A, Jensen TR, Bahl CRH, Dimasi E. Nano size crystals of goethite, a -FeOOH: Synthesis and thermal transformation. J Solid State Chem. 2007;180:1431-5.

[25] Zhou Z, Su M, Shih K. Highly efficient and recyclable graphene oxide-magnetite composites for isatin mineralization. J Alloys Compd. 2017;

[26] Kong D, Wilson LD. Synthesis and characterization of cellulose-goethite composites and their adsorption properties with roxarsone. Carbohydr Polym. 2017;169:282-94.

[27] Taleb K, Markovski J, Hristovski KD, Rajakovic VN. Aminated glycidyl methacrylates as a support media for goethite nanoparticle enabled hybrid sorbents for arsenic removal: From copolymer synthesis to full-scale system modeling. Resour Technol. 2016;2:15-22.

[28] Ramirez-muñiz K, Perez-rodriguez F, Rangel-mendez R. Adsorption of arsenic onto an environmental friendly goethite-polyacrylamide composite. J Mol Liq. 2018;264:253-60.

[29] Ponomar VP. Synthesis and magnetic properties of magnetite prepared by chemical reduction from hematite of various particle sizes. J Alloys Compd. 2018;741:28-34. 
[30] Ganesan V, Lahiri BB, Louis C, Philip J, Damodaran SP. Size-controlled synthesis of superparamagnetic magnetite nanoclusters for heat generation in an alternating magnetic field. J Mol Liq. 2019;281:315-23.

[31] Suppiah DD, Bee S, Hamid A. One step facile synthesis of ferromagnetic magnetite nanoparticles. J Magn Magn Mater. 2016;414:204-8.

[32] Bezdorozhev O, Kolodiazhnyi T, Vasylkiv O. Precipitation synthesis and magnetic properties of selfassembled magnetite-chitosan nanostructures. J Magn Mater. 2016;

[33] Alfredo V, Villegas R, Isaías J et al. Synthesis and characterization of magnetite nanoparticles for photocatalysis of nitrobenzene. J Saudi Chem Soc. 2019;

[34] Jorge M, Nilson M, Machuca-martínez F. Data on the removal of metals (Cr3+, Cr6+, Cd2+, Cu2+, $\mathrm{Ni2+}$ $\mathrm{Zn} 2+)$ from aqueous solution by adsorption using magnetite particles from electrochemical synthesis. Data Br. 2019;24.

[35] Kashif M, Kim Y, Choi Y. Magnetite synthesis using iron oxide waste and its application for phosphate adsorption with column and batch reactors. Chem Eng Res Des. 2019;148:169-79.

[36] Ozcan F, Ersoz M, Yilmaz M. Preparation and application of calix [4] arene-grafted magnetite nanoparticles for removal of dichromate anions. Mater Sci Eng C. 2009;29(8):2378-83.

[37] Kashif M, Phearom S, Choi Y. Chemosphere Synthesis of magnetite from raw mill scale and its application for arsenate adsorption from contaminated water. Chemosphere. 2018;203:90-5.

[38] Marín T, Montoya P, Arnache O, Pinal R, Calderón J. Development of magnetite nanoparticles/gelatin composite films for triggering drug release by an external magnetic field. Mater Des. 2018;152:78-87.

[39] Berger D, Maria C, Ionita D, Valentin I. Box-Behnken experimental design for chromium (VI) ions removal by bacterial cellulose-magnetite composites. Int J Biol Macromol. 2016;(Vi).

[40] Selim MS, Elmarakbi A, Azzam AM, Shenashen MA. Progress in Organic Coatings Eco-friendly design of superhydrophobic nano-magnetite/silicone composites for marine foul-release paints. Prog Org Coatings. 2018;116(February 2017):21-34.

[41] Ding C, Cheng W, Nie X, Yi F. Synergistic mechanism of U (VI) sequestration by magnetite-graphene oxide composites: Evidence from spectroscopic and theoretical calculation. Chem Eng J. 2017;324:11321.

[42] Shokrollahi H. A review of the magnetic properties, synthesis methods and applications of maghemite. J Magn Magn Mater. 2017;426(July 2016):74-81.

[43] Patra D, Gopalan B, Ganesan R. Direct solid-state synthesis of maghemite as a magnetically recoverable adsorbent for the abatement of methylene blue. J Environ Chem Eng. 2019;7(5):103384.

[44] Ianoș R, Moacă E, Căpraru A, Lazău R, Păcurariu C. Maghemite, Y-Fe2O3, nanoparticles preparation via carbon-templated solution combustion synthesis. Ceram Int. 2018;

[45] Dyer L, Fawell PD, Newman OMG, Richmond WR. Synthesis and characterisation of ferrihydrite/silica co-precipitates. J Colloid Interface Sci. 2010;348(1):65-70.

[46] Pariona N, Camacho-aguilar KI, Ramos-gonzález R, Martinez Al, Herrera-trejo M, Baggio-saitovitch E. Magnetic and structural properties of ferrihydrite/hematite nanocomposites. J Magn Magn Mater. 2016;406:221-7. 